\title{
El pensamiento de Pierre Bourdieu: Apuntes para una mirada arqueológica
}

\author{
William R. Fowler \\ Antropólogo. \\ Universidad deVanderbilt, E.E.U.U. \\ Eugenia Zavaleta Lemus \\ Egresada de la Licenciatura en Antropología \\ Sociocultural. \\ Universidad de El Salvador
}

Pierre Bourdieu es uno de los teóricos contemporáneos de la acción social más estudiados en diferentes campos de las ciencias sociales, porque sus postulados invitan a la superación de las antinomias o los dualismos clásicos de la sociología tradicional (objetivismo/subjetivismo, estructura/agencia, individuo/ sociedad, materia/idea, micro/macro, cuali/cuanti) y porque se ha identificado con la superación de los determinismos y sustancialismos, construyendo enfoques más relacionales y procesuales. Sus principales influencias teóricas provienen del marxismo, del estructuralismo francés, de las filosofías de Martin Heidegger, Edmund Husserl, Maurice Merleau-Ponty, Raymond Aron y Ludwig Wittgenstein; inclusive de la tradición epistemológica francesa, que enfatiza el no-positivismo y antievolucionismo. Es, junto con Anthony Giddens, calificado como uno de los dos teóricos de la práctica más influyentes del siglo XX. Bourdieu ha producido una "ontología de las prácticas sociales que busca superar a nivel metodológico y analítico los dualismos de la física y la fenomenología social", y que constituye una "síntesis superadora" de los debates sustancialistas y que abona igualmente al análisis teórico como al empírico (Jaramillo, 2011:413). Esta ontología descansa en categorías sociológicas potentes como habitus, campo, dualidad de la estructura e integración social y sistémica.

Para Bourdieu $(1977,1990)$, la sociedad existe bajos dos formas: los campos (instituciones), que conforman las estructuras sociales externas, y los habitus (disposiciones) que constituyen la organización interna de los agentes (Téllez Iregui, 2002:15). Bajo la tradición del pensamiento relacional, Bourdieu concibe a la sociedad a partir de la comprensión de su carácter heterogéneo y enfatiza en la necesidad de abordarla como un sistema de campos diferenciales, cuyos espacios de lucha diversos son relativamente independientes y 
autónomos, en contraste con las teorías sociales en que la sociedad es un todo homogéneo, integrado y armónico (Jaramillo, 2011:413, 425). Estos campos de lucha son los escenarios en que se mueven los agentes sociales y desde los cuales establecen relaciones que pueden variar desde la dominación hasta la resistencia.

En este ensayo trataremos de mostrar algunos de los principales debates de la teoría sociológica y la teoría social que enmarcan el contexto del pensamiento de Bourdieu, como de sus categorías de análisis. Después, presentaremos las categorías y conceptos bourdieusianos que pueden explicar los procesos de diferenciación y transformación social con el objetivo de señalar su utilidad en la teoría arqueológica. Por último, presentaremos un estudio de caso arqueológico tomado de las investigaciones recientes del sitio arqueológico Ciudad Vieja, El Salvador.

\section{Temas de debate de la teoría sociológica y la teoría social.}

Para entender mejor el aporte de Bourdieu, ofrecemos unos comentarios breves sobre unos temas de debate en la teoría sociológica y la teoría social. Desde la década de 1980 los antropólogos y los arqueólogos han adoptado una serie de preocupaciones teóricas postestructuralistas, desarrolladas en las ciencias sociales, que se centran en la agencia, la estructura; la estructuración y la práctica en conjunción con los problemas relacionados con la acción individual y el individuo; la interacción entre la acción y las estructurassociales, la construcción del ser social, la intencionalidad, la indeterminación; la facultad de actuar y de la dominación y la resistencia. Los enfoques que se ocupan de estas cuestiones se han referido, en diversas ocasiones y con frecuente ambigüedad, a la "teoría de la agencia", la "teoría de la estructuración" y la "teoría de la práctica”.

Antes de examinar estos términos, es necesario evaluar el concepto básico de agencia, su concepto complementario estructura y su uso en la antropología y la arqueología. El concepto de agencia se relaciona con las ideas sobre la personalidad, la voluntad, la libre determinación y la naturaleza de la conciencia. La herencia intelectual de estas ideas se remonta a los filósofos griegos, especialmente Aristóteles, y jugaron un papel importante en el pensamiento de la Ilustración filosófica de Descartes, Hobbes, Locke, Rousseau, Vico, Montesquieu, Hume y Kant. Estas ideas básicas han sido preocupaciones centrales de la ciencia social moderna y son temas importantes en las obras de Marx, 
Spencer, Weber, Durkheim y Mauss. En la teoría sociológica contemporánea aparecen frente a la hegemonía del estructuralismo y el funcionalismo del siglo XX, como el análisis a microescala de las acciones y decisiones de los individuos que se consideran como consecuencias directas de la estructura a macroescalaen, por ejemplo, la etnometodología de Harold Garfinkel (1967), el análisis situacional de ErvingGoffman (1961) y la fenomenología de Alfred Schütz (1967). Este trabajo pone en primer plano la acción individual y subraya la interacción cara a cara, el énfasis en el sentido más bien que la función y en la experiencia vivida. El análisis a microescala ha tenido una fuerte influencia en el trabajo de los teóricos postestructuralistas de la agencia y la práctica.

El término agencia se ha utilizado de manera confusa e imprecisa en la arqueología contemporánea por diferentes analistas que emplean su propia interpretación del término (Dornan, 2002:304). Ian Hodder (2000:22) señala que a principios de la década de 1980 se utilizaba el término agencia en oposición al término comportamiento, con énfasis en la acción individual, la intencionalidad, y la indeterminación en comparación con el conductismo y el determinismo estructural de los sistemas de gran escala y los procesos estudiados por la arqueología procesual (Binford 1962, 1965). El término agencia se convirtió en una palabra clave para hacer frente a la acción individual. Pero a finales de la década de 1980, se hizo evidente que los individuos son difíciles, si no imposibles, de identificar en contextos arqueológicos, y el énfasis en el individuo pasó a centrarse en la "capacidad de actuar", basada en el control de los recursos estratégicos, como bienes de prestigio o conocimiento esotérico (Odre, 2000:22-23; véase también, Jonson, 1989:189-191).

En términos más amplios, todas estas preocupaciones pueden reducirse al debate entre el sustancialismo y el relacionalismo para poder explicar la integración de los conceptos de estructura y gente presentes en los trabajos de Bourdieu. El sustancialismo toma al elemento de análisis (por ejemplo, estructura, agente, sociedad) como una esencia o unidad básica inmutable que, a pesar de su interacción con las demás, sigue teniendo completa independencia como entidades estáticas. El enfoque relacional hace referencia a las unidades de análisis como unidades dependientes entre sí, en simbiosis continua, y cuyo sentido se alcanza al comprenderlas "insertas en configuraciones relacionales y transaccionales y no como entidades discretas predeterminadas en el análisis social" (Jaramillo, 2011:414; véase también, Bourdieu, 1997:115); es decir, no son anteriores a las relaciones que establecen entre sí, sino que se definen por ese mismo proceso (Emirbayer, 1997). 
Bajo esta misma dimensión, uno de los aportes centrales de Bourdieu es la visión del poder como un "recurso" o un "esquema", y no solamente como propiedad de los agentes, el cual se desenvuelve "en relaciones de fuerza, intereses y posiciones" y organiza la sociedad en una jerarquía que los propios agentes legitiman (Jaramillo, 2011:415). Bajo este enfoque, el poder ya no se posee: se construye de forma colectiva. Asimismo, la disociación entre el concepto de estructura social y el de cultura encuentra un punto de fusión. La estructura social ya no es concebida de forma sustantiva, como entramados de sistemas materiales constreñidores desde los que se establece una relación de dominación entre esa estructura y el individuo social; sino como redes de intercambios e interacciones que mantienen lazos de continuidad y permanencia. La noción de estructura de Bourdieu implica una idea flexible de determinación, que reside en el habitus, del que hablaremos más abajo.

La cultura es más que un sistema de normas y valores individuales, producto de experiencias particulares; la nueva propuesta de los autores relacionales es que se conforma a partir de sistemas de "comunicación y narrativas" (Ibíd), de interpretaciones y lenguajes. La fusión que hace Bourdieu de ambas concepciones, disociadas tradicionalmente, resulta en una estructura social que configura las prácticas sociales, los papeles, las normas y la dominación en cuanto se fusiona a partir de las interpretaciones de los sujetos sociales, en donde es menester entender los niveles de constreñimiento en las prácticas sociales a partir de la incorporación de diversos significados. En este sentido, la definición de la estructura social trasciende la figura de un aparato externo al individuo, cuyo carácter es únicamente coercitivo y, a la vez, las prácticas culturales dejan de ser productos de la subjetividad. Es esta fusión o imbricación la que permite que Bourdieu se sitúe como uno de los pensadores que supera los dualismos de la sociología clásica (Ibíd.:415-416).

Además, se puede observar-como destaca Swartz (2012) - , que el pensamiento de Bourdieu es clave para hacer una sociología del poder, con especial atención a las formas de dominación. En efecto, el análisis del poder está en el corazón de la sociología de Bourdieu. Como teórico del conflicto, hace hincapié en el carácter competitivo y estratificado de los mundos sociales, los cuales están firmemente ordenados por los mecanismos y procesos de dominación y reproducción. Propone una teoría del poder simbólico, la violencia y el capital que hace hincapié en el papel activo que desempeñan las formas simbólicas como recursos que constituyen y mantienen las jerarquías sociales. La perspectiva de Bourdieu se opone a la opinión generalizada de que el 
poder simbólico es simplemente simbólico. Su sociología nos sensibiliza a las formas más sutiles e influyentes de poder que operan a través de los recursos culturales, de las categorías simbólicas y las clasificaciones que entretejen la vida cotidiana con las disposiciones institucionales vigentes. Rechazando el reduccionismo económico, Bourdieu identifica una amplia gama de recursos valiosos más allá de los intereses materiales transparentes que funcionan como recursos de poder, que él llama "formas de capital", como el capital social y el capital cultural. Además, las personas y los grupos luchan sobre la definición y distribución de estos capitales en distintos escenarios de poder que Bourdieu llama campos.. Él ve las concentraciones de diversas formas de capital en áreas específicas de lucha, como el campo de poder, el campo político y el campo estatal. La sociología de Bourdieu ofrece herramientas conceptuales para el análisis de tres tipos de poder: el poder conferido a determinados recursos (capitales), el poder concentrado en los ámbitos específicos de lucha en torno a las formas de capital (campos de poder) y el poder práctico, que se da por sentado en la aceptación de las jerarquías sociales existentes (poder simbólico y violencia) (Swartz, 2012:21).

\section{Los conceptos básicos de Bourdieu.}

Bourdieu instituye tres categorías de análisis como herramientas para pensar en la producción y la reproducción del sentido de las prácticas sociales que permiten compararse y aplicarse a las diversas sociedades, aunque sus teorizaciones hayan surgido principalmente a la luz del estudio de la sociedad francesa. Estas categorías, de las que se deslinda todo su análisis, son el habitus, los campos sociales y el capital. Pero esta tríada no puede entenderse si no hablamos del espacio social.

El espacio social global es la sociedad, constituida por campos que se intersectan, integrándola. Es un "conjunto de posiciones distintas y coexistentes, externas unas a otras, definidas en relación unas de otras por su exterioridad mutua y por relaciones de proximidad, de vecindad o de alejamiento y asimismo por relaciones de orden" (Bourdieu, 1997:16), entendida como una distancia social que ordena y jerarquiza. Es una "realidad intangible, que no se puedemostrar ni tocar con el dedo, y que organiza las prácticas y las representaciones de los agentes" (Ibíd.:21-22). En este sentido, la posición que ocupa un actor social en el espacio es congruente con su visión del mundo, con las distintas representaciones que puede hacerse de su propia realidad como de las de los demás agentes. 
El habitus es el cuerpo socializado; está constituido por un sistema de disposiciones, actitudes o inclinaciones adquiridas, duraderas y transferibles que generan y clasifican acciones, percepciones, sentimientos y pensamientos en los agentes sociales de una cierta manera, generalmente escapando a la conciencia y a la voluntad. Estas disposiciones son incorporadas o interiorizadas por los individuos desde su infancia mediante un proceso de socialización multiforme y prolongada. En este último sentido, habitus es "historia hecha cuerpo" (Téllez Iregui, 2002:15, 58).

Se entiende que habitus es la subjetividad socializada y a la vez la interiorización de la exterioridad, o sea, la interiorización de la posición social del agente en su vida cotidiana (y, por ende, la composición de sus capitales): es el "principio generador y unificador que retraduce las características intrínsecas y relacionales de una posición en un estilo de vida unitario, es un conjunto unitario de elección de personas, bienes y prácticas" (Bourdieu, 1997:19). De ahí que Bourdieu haga énfasis en la idea de los cuerpos estructurados: "Si el campo es el marco, el habitus es el efecto del marco, su interiorización: ... es un cuerpo socializado, un cuerpo estructurado, un cuerpo que se ha incorporado a las estructuras inmanentes de un mundo o de un sector particular de este mundo, de un campo, y que estructura la percepción de este mundo y también la acción en este mundo" (Aguirre, 1997). En este sentido, son tan importantes las preferencias y gustos de los agentes (actividades deportivas, preferencias culinarias, bebidas embriagantes, etc.) como las formas de hacerlo, los límites sociales de lo permitido y lo que no permitido, como de lo que es estéticamente aceptado y lo que no es.

La tercera categoría importante para entender el funcionamiento de los otros conceptos es la idea de los campos sociales. Estos se definen como espacios sociales estructurados de posiciones o puestos jerarquizados, es decir, como sistemas de posiciones donde todos los agentes sociales se encuentran interrelacionados de manera durable y dinámica (TellézIregui, 2002:65). Las relaciones entre los participantes de un campo social pueden ser consensuales, pero por lo general tienen un carácter conflictual y antagónico, que tiene origen en el uso y distribución de las formas de capital (bienes materiales o simbólicos) específicas que circulan en los campos.

No estaría de más recalcar el potencial de competencia y conflicto de los campos sociales: son los escenarios de lucha distribuidos en el espacio social por sus tipos diferenciales de capital, que se imponen a los agentes individuales y con- 
tribuyen a conservar o transformar la estructura social (Bourdieu, 1989:29; 1997:49). Son lugares de producción, consumo y reproducción de representaciones del mundo de los agentes. "Se puede describir al campo social como un espacio multidimensional de posiciones tal que toda posición actual puede ser definida en función de un sistema multidimensional de coordenadas cuyos valores corresponden a los valores de las diferentes variables pertinentes: donde los agentes se distribuyen así, en la primera dimensión, según el volumen global de capital que ellos poseen y, en la segunda, según la composición de su capital: es decir, según los pesos relativos de las diferentes especies en el conjunto de sus posesiones" (Bourdieu, 1989:29).

Existen distintos tipos de campos para los agentes: el campo científico, el académico, el religioso, el literario, etc., y cada campo desarrolla sus especies de capital congruentes. Así, cada campo tiene su propia lógica y su propia jerarquía, en donde el agente debe disputarse su lugar según las reglas del juego (Ibíd.::29). Los grupos de agentes que dominan un capital, dominan también el campo de ese mismo capital, y, al reproducirse biológicamente, están efectuando una reproducción social de sus dominios. En este sentido, los espacios sociales, más allá de separar a los agentes según las cuotas de poder acumuladas, agrupan a una cantidad de agentes con estilos de vida similares por tener las mismas propiedades objetivas y las mismas disposiciones habitus), aunque esta aseveración está sometida a la posibilidad de fallas o desfases (Fernández, 2009).

Es justamente esta ubicación de los agentes por medio de campos de dominio en el espacio social (personas con las mismas cuotas de poder, como dinero, círculos sociales, niveles de escolaridad, etc.) la que explica por qué personas de diferente posición social no podrían encontrarse en un punto del espacio social; y si se encontraran, no se entenderían. Los temas de conversación, la elección de los lugares de socialización, las preferencias en una actividad deportiva, en un tipo de música o de películas, está marcada por la distancia social que no permite que se junten personas de diferentes marcos culturales ("límites sociales" de Bourdieu). Al contrario, la proximidad en el espacio social define grupos concretos que no necesariamente constituyen una clase social movilizada, pero que podrían explicar cómo funciona, por ejemplo, el mercado del matrimonio. Así, "la proximidad en el espacio social predispone al acercamiento: las personas inscritas en un sector restringido del espacio estarán a la vez más próximas (por sus propiedades y sus disposiciones, sus gustos y aficiones) y más inclinadas al acercamiento; también resultará más fácil acercarlas, movilizarlas” (Bourdieu, 1997:23). 
El concepto final en el esquema de Bourdieu es el capital, definido como un recurso para posicionarse en el mundo, que permite al actor social ubicarse en un plano de jerarquías y posiciones de menor desigualdad con respecto a agentes más próximos en la escala social. "Representa un poder sobre el campo (en un momento dado) y más precisamente, sobre el producto acumulado del trabajo anterior (en particular, sobre el conjunto de los instrumentos de producción) y por tanto de los mecanismos que aseguran tendencialmente la producción de una categoría particular de bienes, y gracias a ello, a un conjunto de ingresos y beneficios" (Bourdieu, 1989:28). Cada agente es estructura y volumen de capital corporizada y el capital es heredado y adquirido (Bourdieu, 1997:18). A partir de estos principios de diferenciación, los agentes sociales definen sus posturas políticas, sus gustos, deseos y preferencias de consumo (musical, literario, cinematográfico, mascotas, pasatiempos, etc.) y eligen además sus amistades y profesiones.

Las principales especies de capital son el capital económico, el social, el cultural y el simbólico. El capital económico se define por los bienes materiales de producción y de consumo de los agentes. Se puede visualizar en los bienes patrimoniales que los padres acumulan y dejan en heredad a sus hijos como un recurso que los empodera y los distingue en las personas de su generación, y los hace competir por un lugar de renombre en la escala social. La trasferencia de este tipo de capital de una generación a otra demuestra el carácter histórico y mutable de la estructura social. Pero a la vez deja abiertas las posibilidades a los agentes de conservar la estructura, como de cambiarla.

El capital cultural se mide a partir de las habilidades y conocimientos especializados que confieren poder por medio del diploma. El diploma es un tipo de capital cultural institucionalizado por el Estado, que por medio de rituales de consagración separa a los más calificados de los menos, y por ello legitima sus papeles de dominación (Bourdieu, 1997:36-37). Los demás tipos de capital cultural son el objetivado (posesión y uso de bienes culturales como cuadros, máquinas, pinturas, libros, etc.) y el incorporado (hábitos, percepciones y gustos moldeados por los niveles de escolaridad) (Bourdieu, 1979:11-17). El capital social lo constituyen las redes de contactos durables que permiten que los agentes escalen posiciones más convenientes en el espacio social, por ejemplo, los círculos sociales a los que pertenecen los agentes. Y finalmente, el simbólico es el tipo de capital que tiene la capacidad de convertir un capital en otro, por ejemplo, el honor, el prestigio social, etc. Estos tipos de capital pueden ser heredables o adquiridos individualmente. 
El funcionamiento de estos capitales varía según las particularidades de las culturas y las estructuras sociales. En algunas sociedades la conjugación del capital cultural con el capital económico se puede transformar en capital simbólico para configurar la hegemonía de un grupo sobre otro. Sin embargo, puede haber sociedades en que el poder no se detente por la vía de las riquezas materiales o del conocimiento, sino a partir del honor y el prestigio social que deviene de campos más específicos, como el campo del capital religioso o político. Asimismo, también hay sociedades que funcionan con todos los tipos de capital a la vez.

En resumen, la teoría de Bourdieu es tanto una filosofía de la ciencia dedicada a revelar las relaciones objetivas que dan forma a y sostienen la vida social, como una filosofía de acción que toma en cuenta las disposiciones de los agentes, así como las situaciones estructuradas en las que actúan. Esta filosofía de acción se condensa en los conceptos claves de habitus, campo y capital, y se define por la relación de doble vía entre las estructuras objetivas de los campos sociales y las estructuras incorporadas del habitus. La piedra angular del pensamiento de Bourdieu está compuesta por esta relación de doble sentido entre las estructuras objetivas y las estructuras incorporadas (Bourdieu, 1997:8), en donde los espacios sociales son escenarios de producción y reproducción de prácticas y discursos.

\section{El habitus como "estructura estructurante".}

Ya hemos explicado anteriormente las categorías fundamentales que aporta Bourdieu para entender la acción social. Este es un pequeño apartado para describir la formación procesual del habitus, con la intención de extender el diálogo que propone Bourdieu con aquellos conceptos que definen al habitus como "estructura estructurante", superando los determinismos.

Para desarrollar este planteamiento, utilizaremos el trabajo "El espacio social y la génesis de las "clases"” (Bourdieu, 1989) , para entender la formación del habitus como un ejercicio de percepción que combina la estructura social, como una propiedad objetiva de los agentes, con la apreciación individual del mundo social.

Bourdieu propone el habitus como el concepto que sirve para superar la oposición entre el objetivismo y el subjetivismo. Para el objetivismo, los 
agentes estarían determinados por la estructura social, serían meros soportes de la estructura de relaciones en que se encuentran y no efectuarían ningún papel trascendental. Por su parte, las teorías subjetivistas explicarían las relaciones sociales como agregaciones de acciones individuales. De esta manera, si el objetivismo reduce al sujeto a un mero soporte de la estructura, no se puede explicar el hecho de que sujetos de posiciones idénticas produzcan prácticas distintas. Igualmente, los subjetivistas no pueden dar cuenta de las regularidades sociales (que se producen al margen de la voluntad y la consciencia de los individuos). El concepto de habitus da una salida a la cuestión.

El habitus es un sistema de disposiciones incorporadas por los agentes a lo largo de su trayectoria social. Una vez interiorizado el habitus, comenzarán a plasmarse los principios de la arbitrariedad interiorizada, de las estructuras objetivas. Al ser una "estructura estructurante", el habitus se constituye como un esquema generador y organizador de las prácticas sociales y también de las percepciones y apreciaciones de las propias prácticas y las de los demás agentes (Bourdieu, 1989:33; 1997:53-90).

El autor hace especial énfasis en los objetos de percepción y representación del mundo social como objetos históricos, sujetos a las variaciones en el tiempo y a la necesidad de concebirlos como visiones adquiridas y heredables. Al mismo tiempo, esta configuración adquirida está abierta a la variación creativa de los individuos, por tanto, se desprende de un diálogo con los demás agentes que llenan la cotidianeidad de los sujetos sociales: la familia, las colectividades étnicas, de clase o de género.

Estas categorías de percepción se logran a través de la experiencia del mundo social y del trabajo de representación que se da en el agente "más cerca de un inconsciente de clase que de una conciencia de clase, en el sentido marxista" (Bourdieu, 1989:34). La construcción de las representaciones del mundo social "implica una aceptación tácita de su posición", de la "incorporación de las estructuras objetivas del espacio social", y por consiguiente, de las distancias sociales que se deben marcar. Es decir, en vez de formarse una fuerza antagonista de clase, lo que se da es un "principio de realidad más rigurosamente impuesto", que refuerza la identidad de los dominados en relación con los demás sectores sociales (Ibíd).

Este reconocimiento subjetivo de las diferencias tiende a reflejarse en elementos más visibles de la cultura: los espacios de residencia y de acceso 
y movilización de las distintas formas del capital, como su transferencia y permanencia en las estructuras jerárquicas existentes. Esta dominación simbólica permite que grupos de las mismas condiciones materiales se agrupen entre sí, por el hecho mismo de que comparten tanto "propiedades visibles parecidas" como las disposiciones individuales. Como producto del trabajo de percepción, estas uniones se traducen en agrupamientos durables, dotados de significados colectivos. Las interpretaciones del orden social en el que los sujetos sociales están insertos y restringidos es congruente con su posición social.

Es de este modo, particularmente a través de las propiedades y sus distribuciones, que el mundo social logra objetivamente el estatuto de sistema simbólico que, a la manera de un sistema de fonemas, se organiza según la lógica de la diferencia, desviación diferencial, así constituida como distinción significante. El espacio social y las diferencias que emergen "espontáneamente" dentro de él tienden a funcionar simbólicamente como un espacio de estilos de vida... (Ibíd ::36).

La diferenciación social entendida como violencia simbólica es la que nos permite hablar de Bourdieu como un sociólogo del poder, pues se ha detenido a estudiar las dinámicas internas de lucha en los distintos campos sociales, desde donde los individuos y grupos tienen papeles de relevancia en sus acciones.

\section{Estudio de caso arqueológico: Ciudad Vieja, El Salvador, la primera villa de San Salvador.}

Bourdieu se cita a menudo en los estudios arqueológicos, pero las referencias a Bourdieu, al igual que a Giddens, suelen ser gestos simbólicos destinados a dar legitimidad a los proyectos. El verdadero Bourdieu, sociólogo interesado en la dinámica del poder y la cultura, raras veces se encuentra en la interpretación arqueológica. La realización de la contribución de Bourdieu a la teoría arqueológica ha sido incompleta, ya que no ha podido aprovechar plenamente todos los conceptos bourdieusianos - la tríada teórica - de habitus, campo y capital. En particular, la comprensión del habitus queda incompleta (y tal vez superficial) si no la vinculamos a las categorías de campos y capital. Debido a esto, nuestra comprensión teórica de habitus a veces se ha separado de la idea central de que los campos, según Bourdieu, son las ubicaciones de las luchas masivas históricas por el poder. 
Como estudio de caso arqueológico presentaremos algunos de los resultados e interpretaciones más significativas del Proyecto Arqueológico Ciudad Vieja de la Vanderbilt University en El Salvador, llevado acabo de 1996 a 2005 (Fowler, 2011). Este sitio representa las ruinas bien conservadas de la villa de San Salvador, fundada en 1525, rápidamente abandonada y refundada en 1528 como ciudad de la conquista española. La villa de San Salvador tuvo una población indígena residente (en su mayoría nahua) que fue tal vez veinte veces mayor en número que la población española. Abandonada alrededor de 1550-1560, sus 25 a 35 años de ocupación abarcan los años cruciales de la época de la Conquista de América Central.

El muy corto lapso de ocupación hace que el sitio sea idóneo para el estudio arqueológico del cambio social durante el período de la Conquista, y especialmente atractivo para estudiar la interacción dinámica entre los españoles y los pueblos indígenas mesoamericanos durante la primera generación después de la fundación de San Salvador. Las evidencias arqueológicas que revelan que este pueblo fue un lugar de residencia para una población indígena muy grande, son muy convincentes. Es probable que esta población haya sobrepasado varios miles de habitantes; un crisol de pueblos mesoamericanos reunidos para apoyar la empresa de la Conquista, al mismo tiempo que intentaban velar por la preservación y protección de su propia identidad cultural y orígenes, o de variados intereses personales. La parte olvidada de la historia de la Conquista, que está empezando a ser rescatada por un pequeño número de antropólogos e historiadores (Akkeren, 2007; Asselbergs, 2008; Escalante, Arce 2001; Matthew, 2004, 2007, 2012; Matthew y Oudijk, 2007; Restall, 2003; Restall y Asselbergs, 2007), es que esta empresa no habría sido posible sin los aliados nativos. Muchos de los aliados indígenas construyeron y habitaron la villa de San Salvador y contribuyeron de muchas maneras para hacer posible la vida humana allí. La importancia del medio ambiente urbano no puede pasarse por alto.

La conquista española de América fue en general una conquista urbana (Aguilera Rojas, 1973, 1994; Sanz Camañes, 2004; Solano, 1990). Tradiciones urbanas europeas e instituciones con raíces históricas muy profundas proporcionan el marco estructural para la conquista y hegemonía colonial. Las estructuras sociales, económicas, políticas y religiosas, y las prácticas se crearon y se recrearon cada vez que se fundaba una nueva ciudad hispanoamericana. En las acciones específicas, también las poblaciones indígenas partic- 

iparon, en colaboración o resistencia, en concierto con los españoles y otros europeos. Tal vez incluso más que los españoles, las poblaciones mesoamericanas de la época de la Conquista eran también las herederas de profundas tradiciones e instituciones urbanas (Bernal García y García Zambrano, 2006; Webster y Sanders, 2001).

Así es cómo Ciudad Vieja ofrece una oportunidad importante para el estudio arqueológico de la dinámica de interacción cultural español-indígena de la época colonial temprana y la aplicación de la teoría de práctica de Bourdieu. Las investigaciones arqueológicas en el sitio han enfatizado los aspectos espaciales de la ciudad, viéndola como un paisaje cultural: destacando la interacción mutua de los diferentes grupos culturales que compartían el terreno. Aproximadamente dos docenas de ciudades españolas se fundaron en América Central durante el período de la Conquista (Meléndez Chavarri, 1977). Muy pocos de ellos han sido investigados arqueológicamente. Ciudad Vieja es el único entre ellos que tiene ocupación colonial temprana intacta y que destaca buena integridad, conservación y facilidad de acceso. Los paisajes de estos asentamientos formaron la matriz espacial dentro de la cual se realizó la práctica social y las relaciones sociales se promulgaron. Estas prácticas y las relaciones sociales son a la vez reflejadas en el plano de la ciudad y la cultura material del sitio.

Las primeras ciudades coloniales españolas en América desempeñaron un papel crucial en la transformación radical del paisaje. Estos pueblos, con pocas excepciones, fueron construidos sobre un plano en cuadrícula por razones de sentido práctico y tradición cultural. La ciudad en cuadrícula, producto estructural del habitus español, simbolizaba la civilización hispana misma y provocaba una declaración propagandística sobre el poder del Imperio, que se traducía en conceptos muy específicos de orden espacial derivado de la estructura social de la Conquista. Este orden espacial incluía espacios sociales y lugares donde se ejercía el poder de los campos políticos, económicos y religiosos. Henri Lefebvre (1991:151), desde una perspectiva marxista, señala que la ciudad en cuadrícula era "un instrumento de producción: una superestructura ajena al espacio original sirviendo como medio político para introducir una estructura social y económica, de tal forma que pueda ganar pie y efectivamente establecer su "base" en una localidad particular". Otros estudiosos destacan que la ciudad en cuadrícula representaba una afirmación ideológica por los conquistadores que actuaban en nombre de la Corona para imponer el orden moral, jurídico y religioso, o "policía” (Kagan, 2000:131-134; Markman, 1978; Ramírez Ruiz y 
Fernández Christlieb, 2006; Robinson, 1989) sobre las poblaciones indígenas. Esta perspectiva a macroescala, vista a partir de los datos históricos, se complementa en nuestro análisis con una perspectiva a microescala local derivada de la investigación arqueológica (Fowler, 2011). El simbolismo de la ubicación de los edificios del gobierno y de la Iglesia en o cerca de la plaza central pone en alto relieve el poder de la espada y la cruz en la imposición de la voluntad imperial española en el ámbito local (Robinson, 1989:165). En Ciudad Vieja tenemos una buena comprensión de la plaza y el diseño de la cuadrícula que sin duda estaba relacionado con las acciones e interacciones de los agentes y grupos que habitaban en la villa. En base a las ideas de Lefebvre, Kagan y otros podríamos proponer que el plano en cuadrícula fue concebido como un instrumento de producción de un espacio de inculcación ideológica. Pero ¿hasta qué punto se entendió el simbolismo espacial de parte de los habitantes indígenas de la misma manera como se pretendía por parte de los españoles?

Para responder a esta pregunta y para comprender Ciudad Vieja en todo su contexto histórico-cultural, empleamos la perspectiva de la teoría de la práctica de Bourdieu, combinada con la dialéctica socioespacial de Lefebvre. Aunque la fundación y mantenimiento de las ciudades de la conquista española estaban formalmente bajo control centralizado y en general siguieron fórmulas prescritas, en la práctica, la gobernación de cada ciudad era muy descentralizada, lo que resultaba en un alto grado de autonomía de comportamiento y agencia para tomar decisiones locales. La práctica espacial local creó aspectos específicos de variables de comportamiento en los primeros poblados coloniales españoles, cada uno de las cuales debe tener su huella arqueológica correspondiente en la práctica diaria, la negociación en el ámbito de la cultura y en la identificación del espacio físico y social (Roser, 2004:136-137; 179-180).

La cuadrícula urbana como una dimensión estructurante de las prácticas de los pobladores de Ciudad Vieja es de gran potencial si se analiza que, más allá del componente ideológico que interesaba introducir a los conquistadores, en palabras de Bourdieu, se da una diferenciación esta vez objetivada en el espacio social, que legitima la dominación de una sociedad sobre otra. La percepción de esta diferenciación materializada incidiría en las interpretaciones del mundo que tuvieron ambas culturas a partir de su interacción diaria en los diferentes espacios de socialización. De ahí que existieran localidades, seguramente alejadas del centro político y económico, que desarrollaron sus 
propios imaginarios de autonomía en relación con el gran régimen colonial, como respuesta a la jerarquía social y a la agrupación entre pares del mismo habitus. Cómo se vivían los acuerdos, negociaciones, puntos de conflicto y de unión entre una sociedad y otra a partir del material arqueológico nos permitirá llegar al hueso duro de la cultura, en donde la producción de bienes culturales es de gran importancia, como la diversidad artística, por ejemplo, de los estilos cerámicos, pues obedecen a usos y gustos estratificados (Card, 2011).

Igualmente, las categorías de Bourdieu también aportan nuevas pistas de análisis para entender el choque cultural que caracterizó a la época, entendido tradicionalmente como la fusión de diferencias históricas, lingüísticas y étnicas que generaron impacto entre la cultura conquistadora y la conquistada. Ya que Ciudad Vieja es un asentamiento propiamente del período de la conquista española y de principios de la época colonial, las categorías de habitus, campo y capital son de gran importancia para entender las dinámicas de poder, violencia, dominación y resistencia. Mientras los encomenderos se disputaban títulos de nobleza que los harían escalar posiciones de renombre bajo el permiso y autoridad de la Corona española (Sanchíz Ochoa, 1976, 1989; White, 1994), las sociedades prehispánicas no manejaban en su entramado cultural la importancia del capital cultural. Al contrario, fueron sociedades en que los capitales políticos y religiosos, como formas de capital simbólico, mantenían la hegemonía y el orden social por medio del monopolio de bienes de salvación, la recaudación de tributos y del control de las fuerzas militares. El choque cultural, entendido durante y después de la Conquista, es, entonces, un tema susceptible de ser estudiado bajo los conceptos del pensamiento bourdieusiano.

\section{Referencias bibliográficas}

Aguilera Rojas, Javier. Urbanismo español en América. Editora Nacional, Madrid. 1973.

Fundación de ciudades hispanoamericanas. Editorial Mapfre, Madrid. 1994.

Aguirre, Joaquín María Pierre Bourdieu. Razones prácticas: Sobre la teoría de la acción. Documento electrónico, http://www.ucm.es/info/especulo/numero6/bourdieu.htm. Universidad Complutense de Madrid, España. Consultado el día 6 de febrero de 2013. 1997.

Akkeren, Ruud van. La visión indígena de la Conquista. Serviprensa, Guatemala City. 2007. 
Fowler, W. y Zavaleta, E. El pensamiento de Pierre Bourdieu: apuntes para una mirada arqueológica. Pág. 117-135.

Asselbergs, Florine G. L.. Conquered Conquistadors:The Lienzo de Quauhquechollan, a Nahua Vision of the Conquest of Guatemala. University Press of Colorado, Boulder. 2008.

Bernal García, María Elena y Angel Julián García Zambrano.

El altepetl colonial y sus antecedentes prehispánicos: Contexto teórico-historiográfico. In Territorialidad y paisaje en el altepetl del siglo XVI, edición deFederico Fernández Christlieb y Angel Julián García Zambrano, págs. 31-113. Fondo de Cultura Económica, Universidad Nacional Autónoma de México, Mexico City. 2006.

Binford, Lewis R. Archaeology as Anthropology. American Antiquity 28:217-225. 1962.

Archaeological Systematics and the Study of Cultural Process. American Antiquity 31:203-210. 1965.

Bourdieu, Pierre. Outline of a Theory of Practice.Cambridge University Press, Cambridge. 1977.

Los tres estados del capital cultural. Revista Sociológica UAM-Azcapotzalco 5:11-17. 1979.

Distinction: A Social Critique of the Judgement of Taste. Harvard University Press, Cambridge. 1984.

The Social Space and the Genesis of Groups. Theory and Society 14:723-744. 1985 .

El espacio social y la génesis de las “clases.” Estudios sobre las Culturas Contemporáneas, Época I, 3(7):27-55. Universidad de Colima, México. 1989.

The Logic of Practice. Stanford University Press, Stanford. 1990.

Razones prácticas. Editorial Anagrama, Barcelona. 1997.

Habitus. En Habitus: A Sense of Place, edición de Jean Hillier y Emma Rooksby, págs. 27-34. Ashgate Publishing, Aldershot, Inglaterra. 2002.

Bourdieu, Pierre y Loïc, Wacquant

Una invitación a la sociología reflexiva. Siglo XXI Editores, Buenos Aires. 2008.

Brubaker, Rogers

Rethinking Classical Theory: The Sociological Theory of Pierre Bourdieu. Theory and Society 14:745-775. 1985. 
Fowler, W. y Zavaleta, E. El pensamiento de Pierre Bourdieu: apuntes para una mirada arqueológica. Pág. 117-135.

Card, Jeb J. Transformaciones de identidad en El Salvador en la época colonial temprana: La gente y cerámica de la villa de San Salvador en el siglo XVI. La Universidad 1415:245-282. 2011.

Castón Boyer, Pedro. La sociología de Pierre Bourdieu. Revista Española de Investigaciones Sociológicas 76:75-97. 1996.

Dornan, Jennifer L. Archaeology and Archaeology: Past, Present, and Future Directions. Journal of Archaeological Method and Theory 9:303-329. 2002.

Emirbayer, Mustafa. Manifesto for a Relational Sociology. American Journal of Sociology 103:281-317. 1997.

Escalante Arce, Pedro Antonio. Los tlaxcaltecas en Centro América. Consejo Nacional para la Cultura y el Arte, San Salvador. 2001 .

Fernández, Mariano. Bourdieu, Giddens, Habermas: Reflexiones sobre el discurso y la producción de sentido en la teoría social. Cuadernos de H Ideas 3(3). Documento eléctronico: http://perio.unlp.edu.ar/ojs/index.php/cps/article/viewFile/1390/1183. Universidad Nacional de la Plata, La Plata, Argentina. Consultado el día 14 de febrero de 2013. 2009.

Fowler, William R. Ciudad Vieja: Excavaciones, arquitectura y paisaje cultural de primera villa de San Salvador. Secretaría de Cultura de la Presidencia/Editorial Universitaria, San Salvador. 2011.

Garfinkel, Harold. Studies in Ethnomethodology. Prentice-Hall, Englewood Cliffs, New Jersey. 1967.

Goffman, Erving. The Presentation of Self in Everyday Life. Anchor Doubleday, Nueva York. 1959.

Gorski, Philip S. Introduction: Bourdieu as a Theorist of Change. En Bourdieu and Historical Analysis, edición de Philip S. Gorski, págs.1-15.Duke University Press, Durham, North Carolina. 2012.

Hodder, Ian. Agency and Individuals in Long-Term Processes. In Agency in Archaeology,edición de Marcia-AnneDobres y John E. Robb, págs. 21-33. Routledge, Nueva York. 2000.

Jaramillo Marín, Jefferson. Bourdieu y Giddens: La superación de los dualismos y la ontología relacional de las prácticas sociales. Revista CS 7:411-430. 2011. 
Fowler, W. y Zavaleta, E. El pensamiento de Pierre Bourdieu: apuntes para una mirada arqueológica. Pág. 117-135.

Johnson, Matthew H. Conceptions of Agency in Archaeological Interpretation. Journal of Anthropological Archaeology 8:129-211. 1989.

Markman, Sidney D. The Gridiron Town Plan and the Caste System in Colonial Central America. En Urbanization in the Americas from Its Beginnings to the Present, edición de Richard P. Schaedel, Jorge E. Hardoy y Nora Scott Kinzer, págs.471-489. Mouton, La Haya. 1978.

Matthew, Laura E. El náhuatl y la identidad mexicana en la Guatemala colonial. Mesoamérica 40:41-68. 2000.

Whose Conquest: Nahua, Zapoteca, and Mixteca Allies in the Conquest of Central America. In Indian Conquistadors: Indigenous Allies in the Conquest of Mesoamerica. edición de Laura E. Matthew y Michel R. Oudijk, págs. 102-126. University of Oklahoma Press, Norman. 2007.

Memories of Conquest: Becoming Mexicano in Colonial Guatemala. University of North Carolina Press, Chapel Hill. 2012.

Meléndez Chaverri, Carlos. Ciudades fundadas en la América Central en el siglo XVI. Anuario de EstudiosCentroamericanos 3:57-79. 1977.

Orser, Charles E., Jr. Race and Practice in Archaeological Interpretation. University of Pennsylvania Press, Philadelphia. 2004.

Restall, Matthew. Seven Myths of the Spanish Conquest. Oxford University Press, Oxford. 2003.

Restall, Matthew, y FlorineAsselbergs. Invading Guatemala: Spanish, Nahua, and Maya Accounts of the Conquest Wars. Pennsylvania State University Press, University Park. 2007 .

Robinson, David. The Language and Significance of Place in Latin America. En The Power of Place: Bringing Together Geographical and Sociological Imaginations, edición de John A. Agnew and James S. Duncan, págs. 157-184. UnwinHyman, Boston. 1989.

Sanchíz Ochoa, Pilar. Los hidalgos de Guatemala: Realidad y apariencia en un sistema de valores. Publicaciones del Seminario de Antropología Americana, Vol. 13. Universidad de Sevilla, Sevilla. 1976 .

Españoles e indígenas: Estructura social del valle de Guatemala en el siglo XVI. En La sociedad colonial en Guatemala: Estudios regionales y locales, edición de Stephen Webre, 
Fowler, W. y Zavaleta, E. El pensamiento de Pierre Bourdieu: apuntes para una mirada arqueológica. Pág. 117-135.

pags. 33-78. Centro de Investigaciones Regionales de Mesoamérica, Antigua; Plumsock Mesoamerican Studies, South Woodstock, Vermont. 1989.

Sanz Camañes, Porfirio. Las ciudades en la América hispana: Siglos XV al XVIII. Sílex Ediciones, Madrid. 2004.

Schütz, Alfred. The Phenomenology of the Social World. Northwestern University Press, Evanston, Illinois. 1967.

Solano, Francisco de, Ciudades hispanoamericanas y pueblos de indios. Consejo Superior de Investigaciones Científicas, Madrid. 1990.

Swartz, David L. Metaprinciples for Sociological Research in a Bourdieusian Perspective. En Bourdieu and Historical Analysis, edición de Philip S. Gorski, págs.19-35.Duke University Press, Durham, North Carolina. 2012.

TéllezIregui, Gustavo. Pierre Bourdieu: Conceptosbásicos y construcciónsocioeducativa. Universidad Pedagógica Nacional, Bogotá. 2002.

Wacquant, Loïc. Hacia una praxeología social. En Una invitación a la sociología reflexiva, Pierre Bourdieu y LoïcWacquant, págs. 25- 90. Siglo XXI Editores, Buenos Aires. 2008.

Webster, Davidconsciencia y Sanders, William T.

La antigua ciudad mesoamericana: Teoría y concepto. En Reconstruyendo la ciudad maya: El urbanismo en las sociedades antiguas, edición de Andrés Ciudad Ruíz, María Josefa Iglesias Ponce de León y María del Carmen Martínez Martínez, págs. 43-64. Sociedad Española de Estudios Mayas, Madrid. 2001 .

White, Douglas R. Enfoque de redes para el estudio de comunidades urbanas. Estudios Demográficos y Urbanos 9:303-326. 1994. 


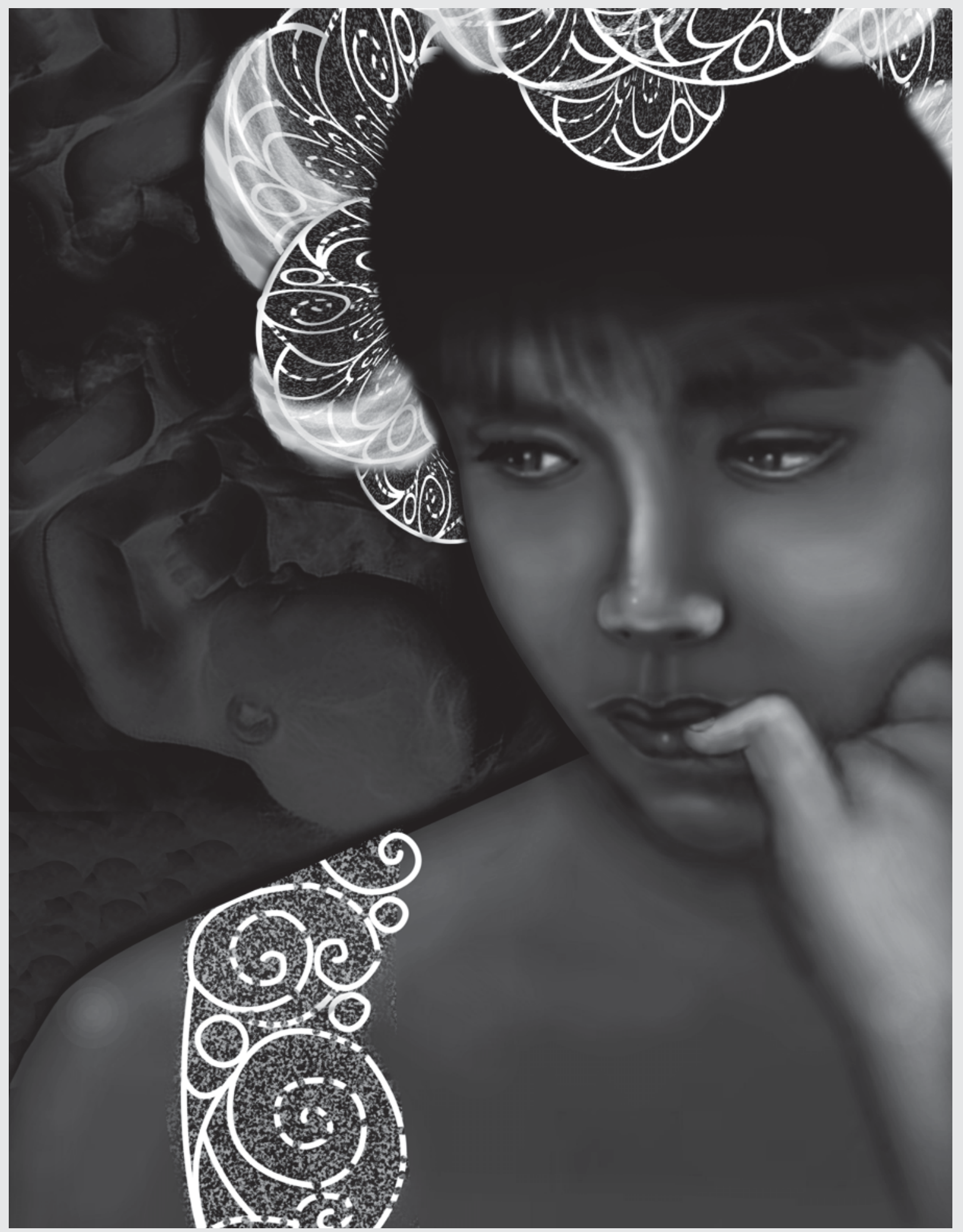

Incertidumbre. Técnica digital, 8.5” x 11". Rita Araujo. 2013. 